\title{
ATTRITION RUPTURES OF FLEXOR TENDONS IN RHEUMATOID ARTHRITIS CAUSED BY BONY SPURS IN THE CARPAL TUNNEL
}

\author{
A Clinical and Radiological Study \\ L. Mannerfelt and O. Norman, Lund, Sweden \\ From the Orthopaedic Clinic, University of Lund
}

The term "attrition" rupture of tendons was coined by Vaughan-Jackson who, in 1948, reported two cases of rupture of extensor tendons through attrition at the inferior radio-ulnar joint. These ruptures were caused by bony irregularities at the distal end of the ulna. Spontaneous ruptures of flexor tendons in rheumatoid arthritis were fully described by Laine and Vainio in 1955.

Pathomechanics of spontaneous tendon ruptures in rheumatoid arthritis-In this disease both flexor and extensor tendons may undergo spontaneous ruptures in five principal ways. 1) Granulomatous rheumatoid tissue invades the tendon along the vincular vessels and in time softens the tendon, which finally ruptures with strain. 2) Occlusion by hypertrophic rheumatoid tissue around the vincular vessels causes localised infarcts in the tendon a few millimetres proximally and distally from the entrance of the vessel. This tendon infarct may in time behave like an intratendinous nodule (Vaughan-Jackson 1968). 3) Rupture may occur from attrition over bony spurs (Vaughan-Jackson 1948, 1962). 4) Spontaneous flexor tendon ruptures may be caused by pressure from the carpal ligament on the already weakened tendons (Straub 1959). 5) Tendons may also rupture after local steroid injections (Moberg 1965).

According to Henderson and Lipscomb (1961) both degeneration by tenosynovitis and fraying of the tendons over adjacent bony structures cause attrition of the tendon. Linscheid and Lipscomb (1962) found that in rheumatoid arthritis the flexor pollicis longus tendon ruptures near its insertion into the distal phalanx. They also noted ruptures of the tendon under the annular ligament or near the point where the tendon joins the muscle. They thought that spontaneous tendon ruptures in rheumatoid arthritis are the result of mechanical attrition of a tendon already weakened by tenosynovial inflammation. Fowler (1963) believed that rupture by attrition of flexor tendons occurred in the vicinity of the hook of the hamate bone and the trapezium. Lipscomb (1965) found that flexor tendons ruptured in the carpal tunnel or within the finger area-that is, in the region where the tendon is surrounded by synovial membrane.

James (1949) presented a case of flexor tendon rupture caused by a sharp bony spicule in a case of Kienböck's disease; the bony spicule had pierced the floor of the carpal tunnel. The case was not regarded as one of rheumatoid arthritis.

We have found no reference in the literature to attrition ruptures of flexor tendons from bony prominences or spurs in the carpal tunnel in rheumatoid arthritis, though Moberg (1965) and Rasmussen and Sneppen (1967) reported both extensor and flexor tendon ruptures.

Distribution of tendon ruptures-The commonest tendon ruptures in patients with rheumatoid arthritis occur in the extensor tendon at the ulnar border of the dorsal aspect of the hand. Next in order of frequency is rupture of the extensor pollicis longus tendon, and least common are those of the flexor tendons. Moberg (1965) reported thirteen cases of spontaneous tendon ruptures in rheumatoid arthritis treated by operation: in seven the rupture was of the flexor pollicis longus and in two the profundus tendon of the index finger was affected. In the remaining four cases rupture was of extensor tendons.

According to Rasmussen and Sneppen (1967) extensor tendons are ruptured more often than flexor tendons. In their series of forty-three cases of tendon rupture, extensor tendons were affected in thirty-six, flexor pollicis longus in three, the profundus tendon of the index 
TABLE I

Distribution and Etiology in Sixty-six Cases of Tendon RUPTURE IN RHEUMATOID ARTHRITIS, 1962 to 1967

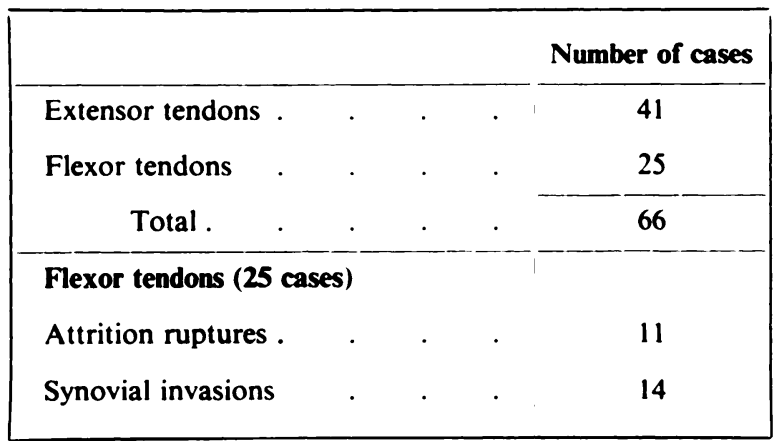

TABLE II

Tendons Affected in Sixty-six Cases of Tendon Rupture in Rheumatoid Arthritis, 1962 to 1967

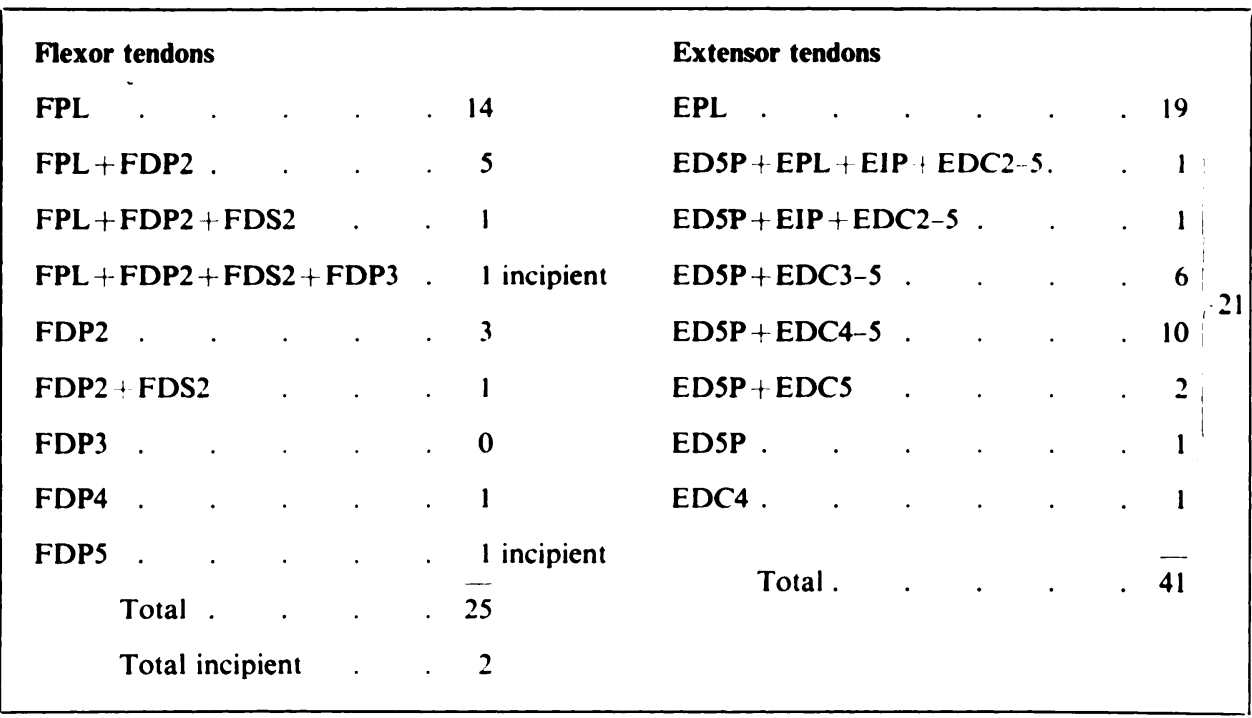

TABLE III

Sex Distribution in Sixty-six Cases of Tendon Rupture IN Rheumatoid ARthritis, 1962 to 1967

\begin{tabular}{|c|c|c|c|c|}
\hline & & Male & Female & Total \\
\hline Number of cases & & 36 & 30 & 66 \\
\hline Extensor tendons & $\cdot$ & 23 & 18 & 41 \\
\hline Flexor tendons & & 13 & 12 & 25 \\
\hline \multicolumn{5}{|l|}{ Attrition ruptures } \\
\hline Flexor tendons & ${ }^{\circ}$ & 2 & 9 & 11 \\
\hline
\end{tabular}

VOL. 51 B, NO. 2, MAY 1969 
finger in two, the abductor pollicis longus in one, and extensor carpi ulnaris in one. Only two of the forty-three ruptures (of the extensor tendon to the third digit) were ascribed to attrition. Sixteen were caused by synovial disease, and in twenty-five instances the cause was unknown.

In these two papers (Moberg 1965 and Rasmussen and Sneppen 1967) there was no discussion of the cause of attrition ruptures of the flexor tendons. It is, however, of interest that these two groups of authors found flexor tendon ruptures to occur more often on the radial side (flexor pollicis longus and the profundus tendon of the index finger) than on the ulnar side.

Bony spicules in rheumatoid arthritis - Lexer (1903) showed how inoculations with staphylococcus aureus followed the vessels into the bone and caused destruction of bone. Moberg, Wassén, Kjellberg, Zettergren, Scheller and Aschan (1966) demonstrated how the granulomatous tissue in rheumatoid arthritis ate away at bony structures just proximal to the articular cartilage. These granulations too follow the vascular pathways. Moberg's findings referred to the heads of the metacarpal bones, but they can no doubt be applied to bones in the carpal region of the hand.

\section{THE PRESENT INVESTIGATION}

Because it has been said that extensor tendon ruptures in rheumatoid arthritis are far more common than flexor tendon ruptures, we decided to study a series of tendon ruptures in rheumatoid arthritis during the years 1962 to 1967 treated at the Rheuma-Centre of Lund. Our three main problems were as follows. 1) To find out the relationship between extensor tendon and flexor tendon ruptures around the wrist during an observation period of six years. 2) To find out possible reasons for ruptures on the flexor aspect of the hand-especially in the carpal tunnel. 3) To investigate a radiological method for exploring the carpal tunnel, especially its floor and walls. This was an imperative task, because the conventional method of demonstrating the carpal tunnel radiologically is seldom possible in advanced rheumatoid arthritis on account of the restriction of wrist movement.

Material-During a period of six years (1962 to 1967) we have collected a series of sixty-six cases of tendon rupture in rheumatoid arthritis, in all of which operation was done. Rather fewer than one-third of the lesions affected the flexor tendons (Tables I and II). The sex distribution was roughly equal in relation to involvement of extensor or flexor tendons (Table III). Thirty-six of the patients were males. Attrition by a bony spur in the carpal tunnel was found in eleven out of twenty-five flexor tendon ruptures. Nearly all these patients were women (Table III).

Pathological considerations-The bony spurs arose from the scaphoid bone or from the trapezium and pierced the floor of the carpal tunnel in relation to the tendon of the flexor pollicis longus. Lexer's (1903) and Moberg et al.'s (1966) findings indicate how these spurs are formed, in the light of the location of the nutrient foramina of the scaphoid bone, especially on the distal volar side. From earlier investigations (Schnek 1930, Lützeler 1932, Grettve 1955) the number, size and position of these nutrient foramina are known. We have examined fifteen navicular bones chosen at random and in all specimens found fairly large nutrient foramina in the distal volar and radial aspects, especially in the hook of the scaphoid bone facing the volar proximal part of the trapezium (Fig. 2). This is completely in line with the findings of Taleisnik and Kelly (1966), who, with their detailed dissecting technique, showed how tenuous vessels ran along the trapezioscaphoid connections to reach the tuberosity of the scaphoid bone. It is therefore obvious that the blood supply to the trapezium and the scaphoid bones is performed by fairly large vessels in the vicinity of the joint between these two bones on the volar lateral aspect.

The flexor pollicis longus is directly related to this region (Fig. 1); because its tendon goes around the hook of the distal part of the scaphoid bone, we have called this region 
" the critical corner." While the rheumatoid process is active the intercarpal ligaments become lax, the carpal bones are subluxed in a volar direction to the distal radius, and the normal anatomy is further disturbed by a volar proximal pull exerted by the carpal flexors. Along the nutrient vessels there is a continuous invasion of destructive rheumatoid granulomatous tissue causing erosions, especially in the distal volar part of the scaphoid bone and in the proximal volar part of the trapezium (Fig. 3). The remaining part between the erosions thus appears as sharp spurs (not to be confused with the hypertrophic spurs seen in osteoarthritis). These sharp spicules finally cut through the weakened floor of the carpal tunnel and in this way lead to attrition of flexor tendons, especially those on the radial side of the carpal tunnel.

According to the literature ruptures of the flexor pollicis longus tendon are infrequent. Nevertheless we strongly believe that these ruptures are more common than has previously been assumed. This is because, even if the tendon has ruptured, continuity still exists through the tube of paratenon (Fig. 4). If there

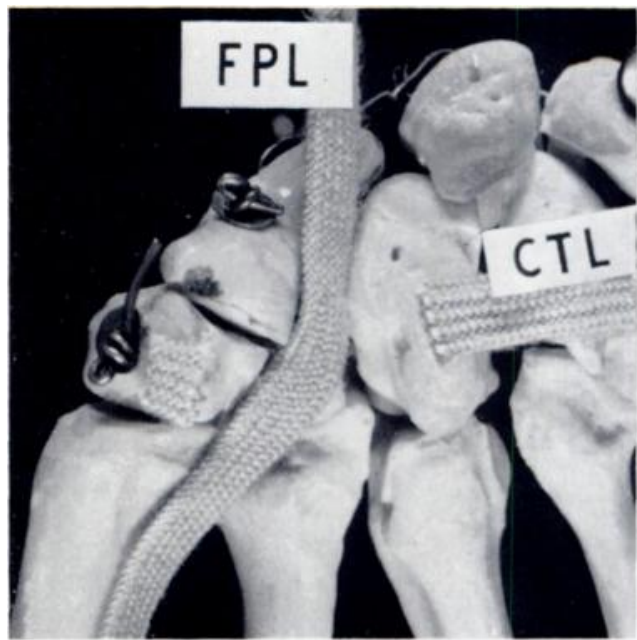
FIG. 1

Model showing relationship between the flexor pollicis longus tendon (FPL) and the ". critical corner" consisting of the distal volar ulnar part of the scaphoid bone and the proximal volar ulnar part of the trapezium. CTL represents remaining part of the flexor retinaculum. is no gross deformity of the interphalangeal joint the patient develops a true though weak power of flexing the distal phalanx. In our twenty-five cases of rupture of flexor tendons in the carpal tunnel the tendons most commonly affected were those on the radial side (Table II). This is in contrast with findings on the extensor surface, where (if the specific rupture of the tendon of the extensor pollicis longus is excluded) the tendons on the ulnar side are more commonly affected. Moreover, the patients can quite often describe how the flexor tendon to the thumb first ruptured and was followed by the tendon of the index finger. In one case we found total ruptures of the following tendons:

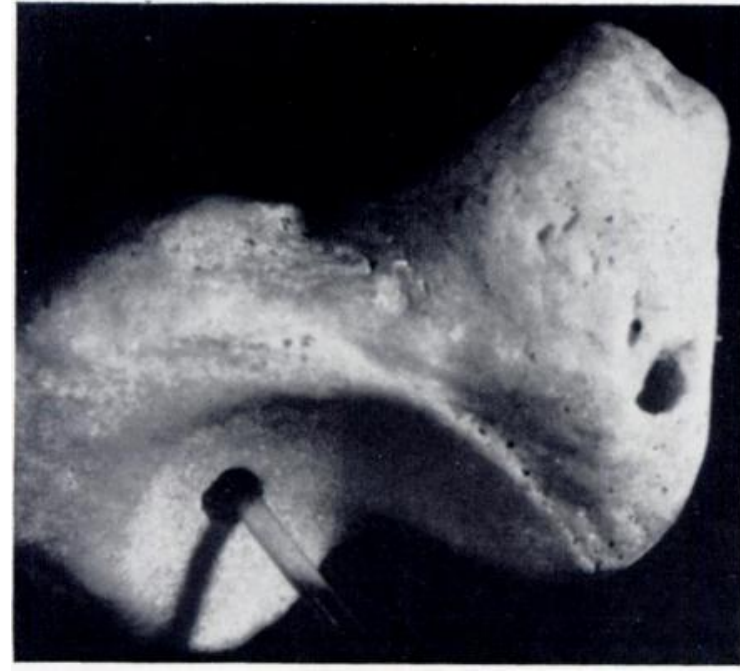

FIG. 2

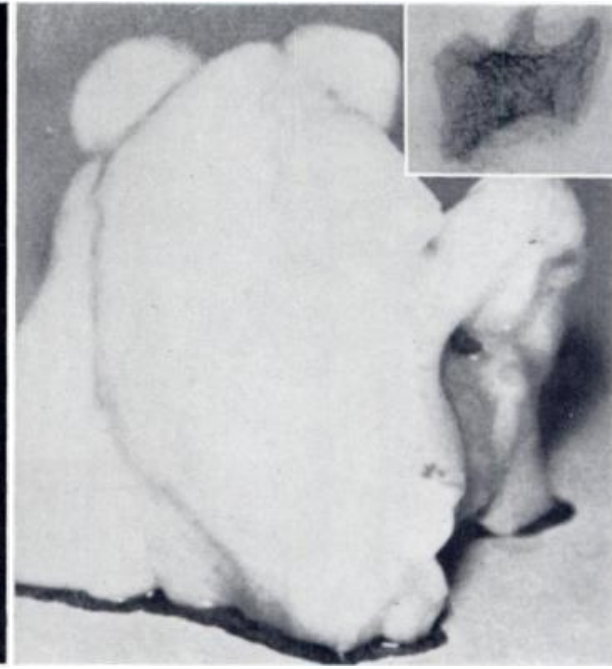

FIG. 3

Figure 2-Large nutrient foramina in distal volar part of left scaphoid bone chosen at random. Figure 3Corresponding part of scaphoid bone of a woman in one of the cases of attrition by a spur, showing the eroded area around the entrance of the nutrient vessels and the spurs representing bone remaining between erosions. Inset shows radiograph of spur. Note the sharp spicules.

VOL. 51 B, NO. 2, MAY 1969 
flexor pollicis longus, flexor digitorum profundus and superficialis of the index finger and also an incipient rupture of the profundus tendon to the middle finger. The flexor tendons lying on the ulnar side were intact. We believe that these ruptures occur consecutively, starting with the flexor pollicis longus and thereafter affecting flexor tendons in a more ulnar direction.

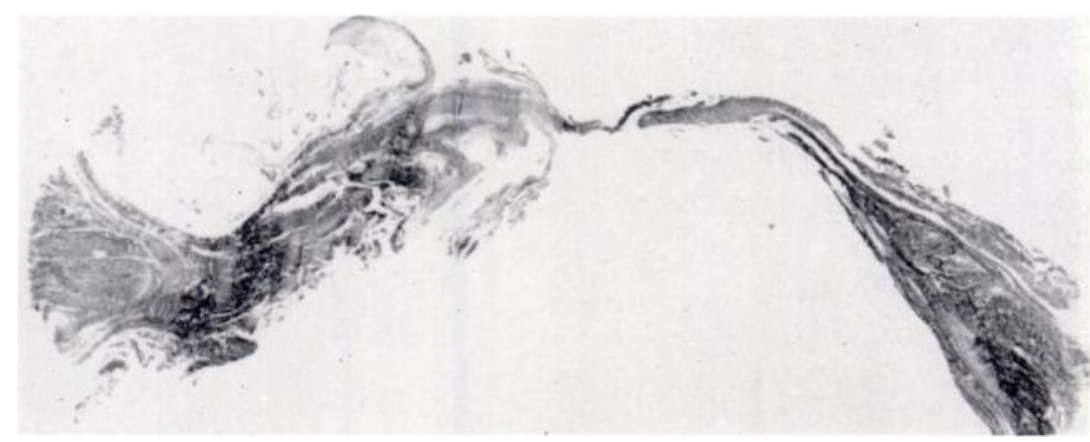

Fig. 4

Longitudinal section of flexor pollicis longus tendon showing attrition rupture. The tendon ends are linked by a tube of paratenon.

Radiographic technique-The radiographic examination is focused on the bone structures along the path of the flexor tendons in the distal as well as the proximal part of the carpal tunnel.

Conventional techniques are as a rule sufficient to show the proximal part, that is, the radiocarpal level. These techniques will admittedly show pathological changes in the distal part of the tunnel but only occasionally will they show them in sufficient detail. In addition, it is often impossible to achieve an axial projection of the carpal tunnel because of stiffness of the wrist. Tomography has been shown to be an excellent method for demonstrating the areas of interest. Because the carpal bones are fairly compact, linear tomography gives a very disturbing linear smear. Another direction of movement is necessary: we have used circular

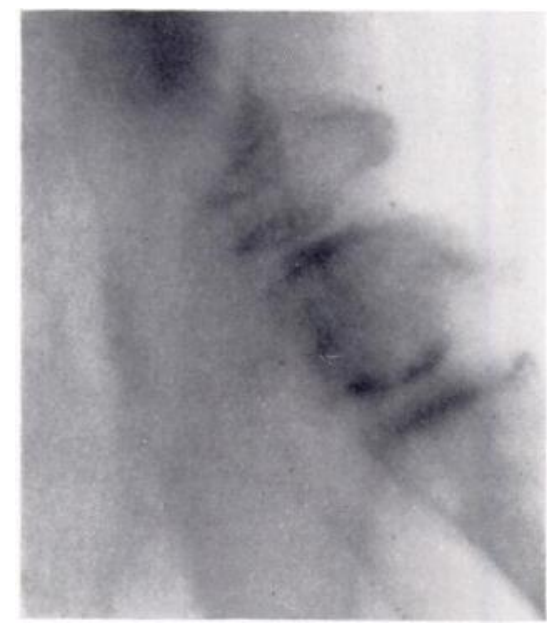

Fig. 5

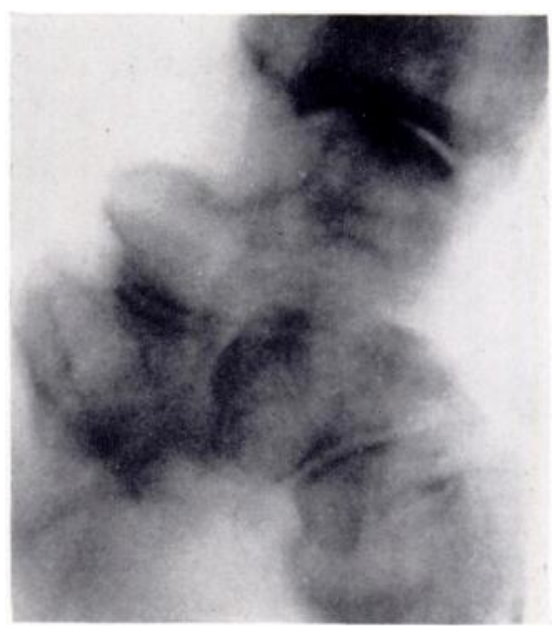

Fig. 6

Tomographic appearances. Figure 5-Frontal projection of trapezium and scaphoid bones. Note multiple erosions and spurs. Figure 6-Lateral projection of the volar aspect and the trapezoid and capitate bone.

tomography with an angle of 42 degrees. In frontal projection the ulnar facet of the trapezium and scaphoid bone, along which the flexor pollicis longus tendon makes a radially concave curve, is recorded (Fig. 5). In this projection an almost axial view of the hook of the hamate bone as well as its counterpart, " the hook" of the trapezium, is also achieved. 
In lateral projection the volar aspect of the scaphoid and capitate bones and of the trapezoid is seen (Fig. 6). This is of special importance in cases of advanced volar sliding of the carpus. The flexor tendons, which in this projection normally have a fairly straight course, now pass over the bulging floor of the carpal tunnel. This leads to a more intimate contact between the tendons and the trapezoid or even the capitate and hamate bones.

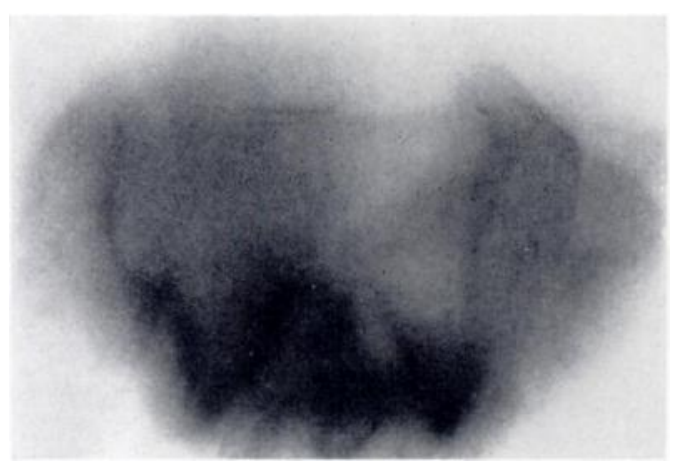

FIG. 7

Axial projection of the carpal tunnel (tomograph).

\section{Fig. 8}

Positioning for axial view of the carpal tunnel in circular tomography. The tomographic plane is perpendicular to the long axis of the forearm.

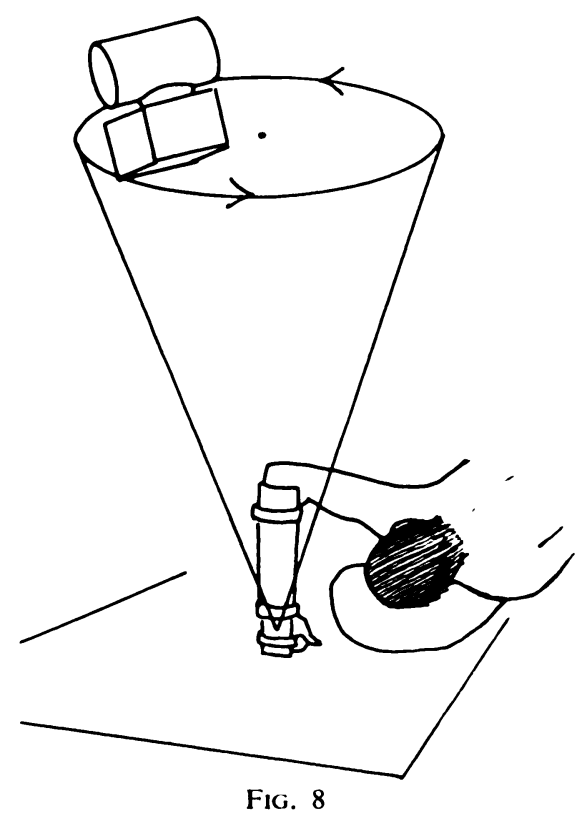

Fig. 8

An axial projection of the carpal tunnel-a picture of a plane perpendicular to the long axis of the forearm - can also be obtained tomographically (Fig. 7). The patient lies prone with the forearm in the boxing position perpendicular to the examining table (Fig. 8). A dorsal splint supports the wrist to the level of the metacarpo-phalangeal joints and prevents movement. In these cuts the floor of the carpal tunnel as well as the opposing aspects of the hook of the hamate bone and of the trapezium can be studied.

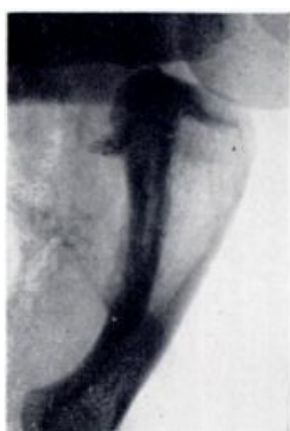

FIG. 9

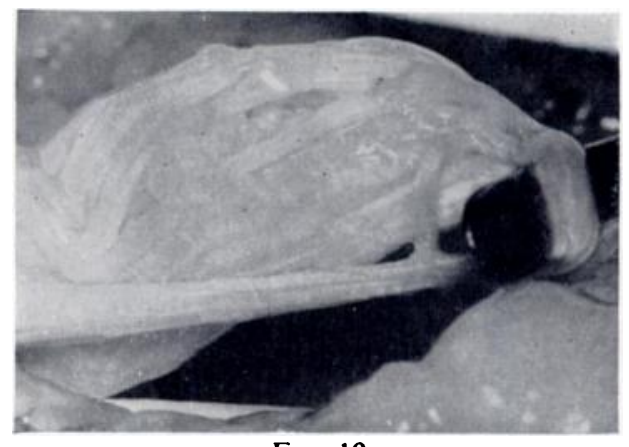

FIG. 10

Figure 9-Radiograph showing exostosis on the volar part of the proximal phalanx. Figure 10-Fraying of superficialis tendon by attrition on the sharp exostosis.

Findings at operation-We have seen how frayed and mechanically damaged are the tendons ruptured by spur attrition in comparison with the tendons ruptured by synovial invasion. The structure of the mechanically destroyed tendon is the same as was seen in James's (1949) case. It is also very much like that seen in another case, where there was an incipient attrition 
rupture of the superficialis and profundus tendons in a patient with generalised cartilaginous exostoses. In this case a radiograph showed a little sharp bony exostosis on the volar aspect of the basal phalanx of the index finger (Fig. 9). The profundus and superficialis tendons were found to be frayed simply by attrition (Fig. 10).

Findings at operation were very much alike in eleven out of twenty-five cases of rupture of the flexor tendons. The volar part of a carpal bone had broken through the floor of the tunnel just proximal to the carpal ligament. These bony spurs were often sharp. Radiographs during operation showed that they arose either from the scaphoid bone or from the trapezium (Figs. 11 and 12). The middle point of the paratenon tube mentioned above was opposite the level of the spur, indicating the origin of the rupture.

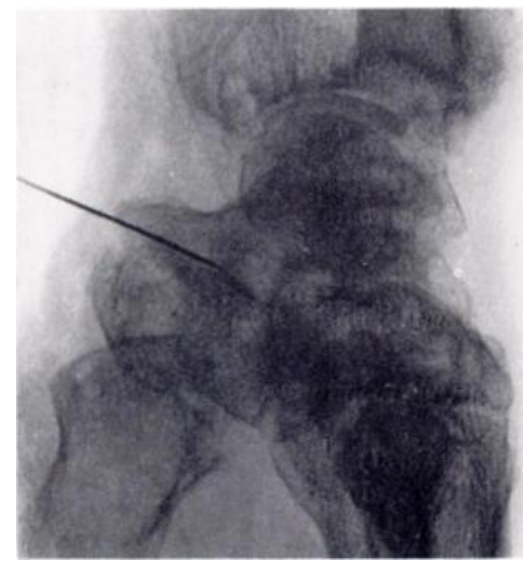

FIG. 11

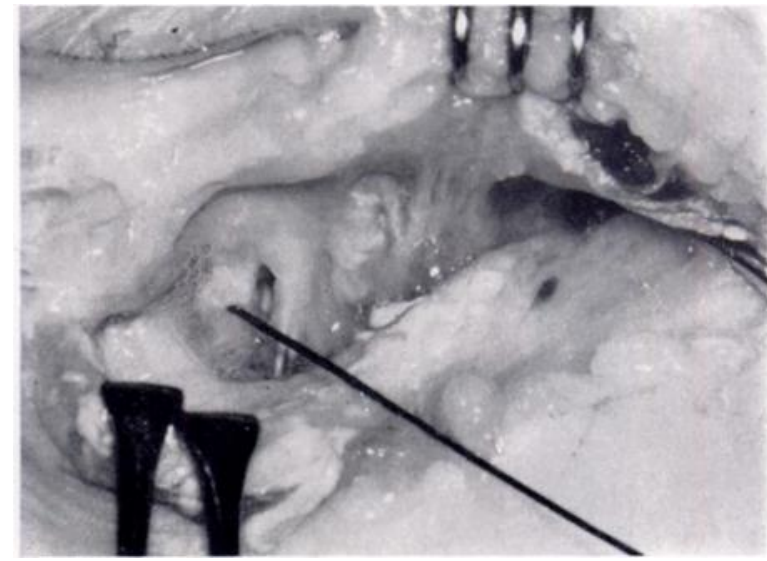

Fig. 12

Figure 11--Radiograph taken during operation. The indicator shows the scaphoid bone piercing the floor of the carpal tunnel. Figure 12-Findings at operation. Note the frayed tendons adjacent to the spur under the double retractors. On the right (distally) can be seen the points of a clamp on the proximal stump of the distal part of the ruptured tendon of the flexor pollicis longus.

Treatment-In cases of rupture of the flexor pollicis longus and profundus tendon of the index finger, repair was done with free tendon grafts or by transfer from an adjacent superficialis tendon. It was not possible to suture any of these ruptured tendons, because of retraction of the proximal part. In long-standing cases it was obvious that the excursion of the flexor pollicis longus muscle was small; nevertheless, several patients later showed fairly good though not strong power of flexion of the thumbs after free tendon grafts.

The value of radiographic examination-We are now able to explore the floor of the carpal tunnel radiographically and to demonstrate bony spurs piercing or about to pierce the floor. In some of the most recent cases we have been able before operation to show a roughened area in the vicinity of the " critical corner." At operation in these cases we found a pre-rupture state of the flexor pollicis longus tendon caused by a spur from the scaphoid bone piercing the floor of the radial side of the carpal tunnel. Only the deep part of tendon was frayed. Removal of the spur and covering of the floor with material from the carpal ligament may have saved these tendons from later attrition rupture.

\section{SUMMARY AND CONCLUSIONS}

1. Bony spurs resulting from erosion of the scaphoid bone and trapezium in rheumatoid arthritis can pierce the floor of the carpal tunnel and cause attrition rupture of flexor tendonsmost often the flexor pollicis longus tendon.

2. It is difficult to show these spurs on conventional radiographs, but using a special tomographic technique we have been able to explore the floor of the carpal tunnel in three 
planes. Using this technique we have been able to guard the flexor pollicis longus against attrition rupture by early excision of a spur from the scaphoid bone.

3. Our findings support the recommendation of free division of the flexor retinaculum in rheumatoid arthritis. When doing this the surgeon should always look for a bony spur piercing the floor of the carpal tunnel.

\section{REFERENCES}

Fowler, S. B. (1963): The Hand in Rheumatoid Arthritis. American Surgeon, 29, 403.

Grettre, S. (1955): Arterial Anatomy of the Carpal Bones. Acta Anatomica, 25, 331.

Henderson, E. D., and Lipscomb, P. R. (1961): Rehabilitation of the Rheumatoid Hand by Surgical Means. Archives of Physical Medicine and Rehabilitation, 42, 58.

James, J. I. P. (1949): A Case of Rupture of Flexor Tendons Secondary to Kienböck's Disease. Journal of Bone and Joint Surgery, 31-B, 521.

LaINe, V. A. I., and VAINIO, K. J. (1955): Spontaneous Ruptures of Tendons in Rheumatoid Arthritis. Acta Orthopaedica Scandinavica, 24, 250.

LeXer, E. (1903): Die Entstehung entzündlicher Knochenherde und ihre Beziehung zu den Arterienverzweigungen der Knochen. Archiv für klinische Chirurgie, 71, 1.

Linscheid, R. L., and Lipscomb, P. R. (1962): Advances in Surgical Treatment of the Rheumatoid Hand. Minnesota Medicine, 45, 273.

LIPSCOMB, P. R. (1965): Surgery of the Arthritic Hand. Mayo Clinic Proceedings, 40, 132.

LüTZeler, H. (1932): Die Entstehungsursache der Pseudarthrose nach Bruch des Kahnbeins der Hand. Deutsche Zeitschrift fiir Chirurgie, 235, 450.

Moberg, E. (1965): Tendon Grafting and Tendon Suture in Rheumatoid Arthritis. American Journal of Surgery: 109, 375.

Moberg, E., Wassen, E., Kuellberg, S. R., Zettergren, L., Scheller, S., and Aschan, W. (1966): The Early Pathologic Changes in Rheumatoid Arthritis. Acta Chirurgica Scandinavica, Supplementum, 357, 142.

Rasmussen, K. B., and SNeppen, O. (1967): Operativ behandlung af polyarthritis. Nordisk Medicin. $77,433$. SCHNEK, F. (1930): Die Verletzungen der Handwurzel. Ergebnisse der Chirurgie und Orthopädie, 23. 1.

Straub, L. R. (1959): The Rheumatoid Hand. Clinical Orthopaedics, 15, 127.

TAleisNiK, J., and Kelly, P. J. (1966): The Extraosseous and Intraosseous Blood Supply of the Scaphoid Bone. Journal of Bone and Joint Surgery, 48-A, 1125.

VAughan-Jackson, O. J. (1948): Rupture of Extensor Tendons by Attrition at the Inferior Radio-Ulnar Joint. Journal of Bone and Joint Surgery, 30-B, 528.

Vaughan-Jackson, O. J. (1962): Rheumatoid Hand Deformities Considered in the Light of Tendon Imbalance. Journal of Bone and Joint Surgery, 44-B, 764.

Vaughan-Jackson, O. J. (1968): Personal communication. 